\title{
New, specific ultrasonographic findings for the diagnosis of pulled elbow
}

\author{
Yu Sung Lee, You Dong Sohn, Young Teak Oh \\ Department of Emergency Medicine, Hallym University Sacred Heart Hospital, Anyang, Korea
}

Objective Among infants and preschool children with complaint of upper extremity immobility, pulled elbow, also known as nursemaid's elbow or radial head subluxation is the most common cause presenting to pediatric emergency departments. However, proper tools to diagnose pulled elbow remain limited. We conducted a study to determine the feasibility of ultrasonography in diagnosing pulled elbow.

Methods Infants and preschool children presenting to an urban emergency department with the complaint of upper extremity immobility between April and July 2013 were enrolled. The following ultrasonographic information was recorded: (1) whether there was a change in the shape of the supinator muscle, (2) whether there was an annular ligament in place, and (3) whether there was an enlargement of the synovial fringe. We used the affected arms' ultrasonographic images as the study group and opposite arms' ultrasonographic images as the control group.

Results When we diagnosed pulled elbow using ultrasonographic findings (i.e., the annular ligament was not in place), we found the following results: sensitivity, $64.9 \%(95 \% \mathrm{Cl}, 47.5 \%$ to 79.8\%); specificity, $100.0 \%$ (95\% Cl, 90.5\% to $100.0 \%)$; positive predictive value, $100.0 \%$ (95\% $\mathrm{Cl}, 85.8 \%$ to $100.0 \%)$; and negative predictive, $74.0 \%(95 \% \mathrm{Cl}, 59.7 \%$ to $85.4 \%)$.

Conclusion A pulled elbow can easily be confirmed by ultrasonography when the annular ligament is displaced.

Keywords Diagnosis; Sensitivity and specificity; Ultrasonography

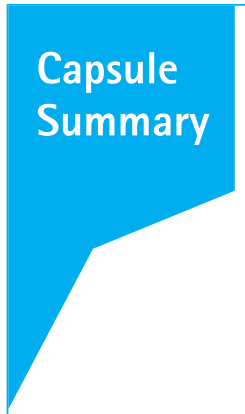

What is already known

Previous studies regarding usage of ultrasonography for pulled elbow was not sufficient to generalize the methods into clinical practice. Generally, the diagnosis of pulled elbow depends on history of mechanism as well as physical examination.

What is new in the current study

Ultrasonographic findings is useful to confirm pulled elbow even with unclear mechanism of injury and inconclusive physical examination.
elSSN: 2383-4625

Received: 27 June 2014

Revised: 18 August 2014

Accepted: 28 August 2014

Correspondence to: You Dong Sohn Department of Emergency Medicine, Hallym University Sacred Heart Hospital, 22 Gwanpyeong-ro 170 beon-gil, Dongan-gu, Anyang 431-796, Korea

E-mail: medyshon@hallym.or.kr

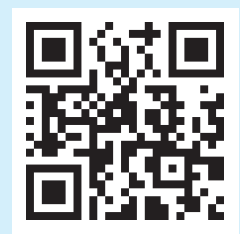

How to cite this article:

Lee YS, Sohn YD, Oh YT. New, specific ultrasonographic findings for the diagnosis of pulled elbow. Clin Exp Emerg Med 2014;1(2):109-113.

This is an Open Access article distributed under the terms of the Creative Commons Attribution Non-Commercial License (http:// creativecommons.org/licenses/by-nc/3.0/). 


\section{INTRODUCTION}

Among infants and preschool children with complaint of upper extremity immobility, pulled elbow, also known as nursemaid's elbow or radial head subluxation was the most common cause presenting to pediatric emergency departments (EDs).' There have been many attempts to diagnose pulled elbow through radiologic findings, but results have been ambiguous. ${ }^{2-4}$ When physicians diagnose pulled elbow, they usually depend on a history, such as passive traction by parents, and on physical examinations. But patients with pulled elbow are too young to get an exact history from and to perform definite physical examination on. For this reason, misdiagnosis of a fracture as a pulled elbow may be possible. One study reported that there were 11 cases of fractures misdiagnosed as pulled elbow over a 36 -month period. ${ }^{5}$

Recently, we introduced a method to diagnose pulled elbow through new ultrasonographic findings. ${ }^{6}$ This study determines the accuracy of ultrasonography in the diagnosis of pulled elbow as a follow-up to our previous investigation.

\section{METHODS}

\section{Study design and setting}

We conducted a retrospective study of patients with suspected pulled elbow who presented to an urban ED with the complaint of upper extremity immobility between April and July 2013.

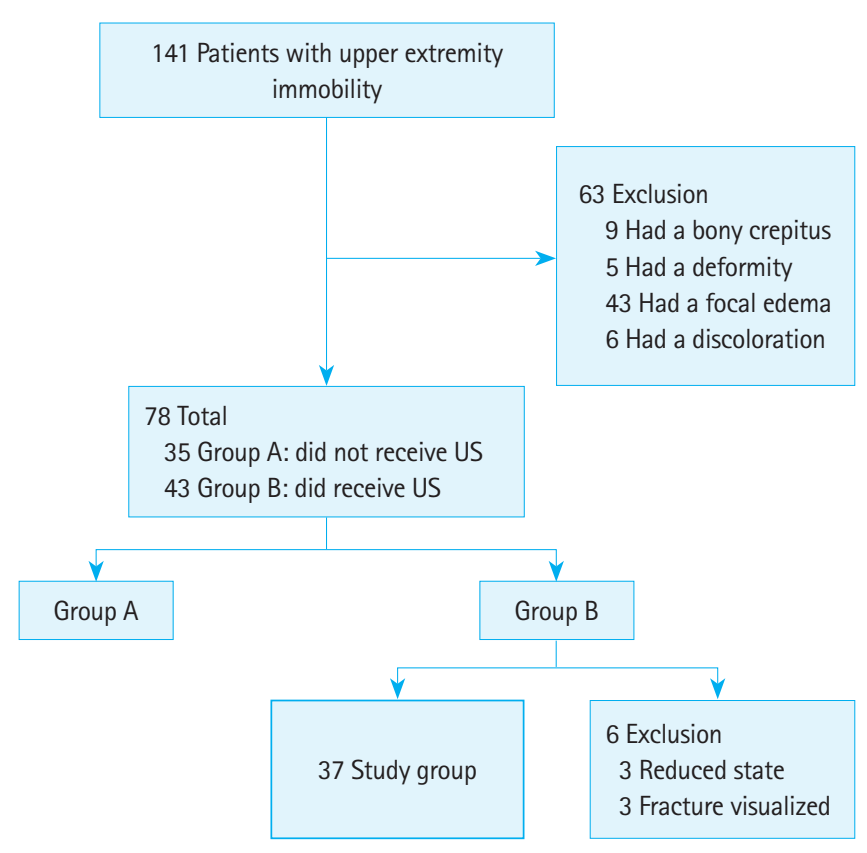

Fig. 1. Flowchart of study inclusion. US, ultrasonography.

\section{Subjects}

We retrospectively reviewed the charts of 141 patients with history, symptoms, and signs suggestive of pulled elbow. The exclusion criteria are: (1) bony crepitus, (2) deformity, (3) focal edema, or (4) discoloration. Of the remaining patients, we collected information on those who underwent ultrasonography; we then analyzed the patients who were confirmed as having pulled elbow (Fig. 1). We categorized the arms as affected and unaffected based on mobility of the upper extremities. Patients were finally diagnosed as pulled elbow, if they could flex, and rotate their affected arms without pain after reduction. No one presented with bilateral upper extremity immobility in our study.

\section{Measures and protocols}

Standardized data collection was performed by trained emergency physicians using formatted case report forms that included patient age, sex, and affected side. We also collected ultrasonographic images of both elbows to compare affected and unaffected elbows. We conducted ultrasonography according to our previous study. ${ }^{6}$ This was performed as follows: the child was held on the parent's lap while the parent was seated opposite the ultrasound operator. The elbow was fully extended, and then a probe was used to locate the anterior portion of the radiocapitellar joint longitudinally (Fig. 2). When the radiocapitellar joint was noted clearly, we recorded: (1) whether there was a change in the shape of the supinator muscle, (2) whether the annular ligament was in place, and 3) whether there was an enlargement of the synovial fringe (Fig. 3). We used the affected arms' ultrasonographic images as the study group and opposite arms' ultrasonographic images as the control group.

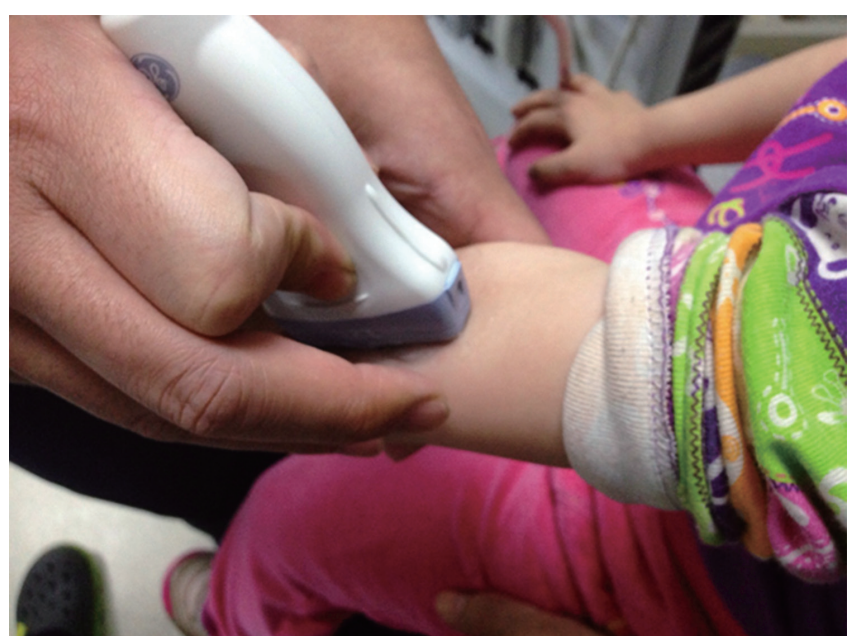

Fig. 2. Position of patients and ultrasound. Elbows fully extended with the probe on the anterior portion of capitellar-radial joint. 
All images were taken by two emergency medicine residents who received an 1-hour training and a 1-hour hands-on practice. All images were validated by one expert physician who was blinded to the patients' histories, including their symptoms and final diagnoses. Ultrasonography was performed using a high-resolution 14-MHz linear probe on a GE S5 ultrasound machine (GE Healthcare, Milwaukee, WI, USA).

\section{Data analysis}

Statistical analyses were performed using MedCalc ver. 12.5 (MedCalc Software, Ostend, Belgium). We described continuous variables using 95\% confidence intervals and binary variables using frequency (\%). We also performed chi-squared tests to determine if there was a statistical difference between the binary variables.

We performed McNemar test to investigate if there was a difference between the paired binary variables. A P-value of 0.05 or less was considered statistically significant. We then calculated the sensitivity and specificity of results with 95\% confidence intervals.

\section{RESULTS}

A total of 141 patients with upper extremity immobility came to our ED during the study period. Of these, 63 were excluded from the study for the following reasons: bony crepitus (9), deformity (5), focal edema (43), or discoloration (6). Of the remaining 78 patients, 43 were examined with ultrasonography. Three patients were excluded because of fracture and the other three were excluded because they arrived at the ED after elbow was self-reduced. Finally, we studied 37 patients (Fig. 1). The mean age was $28.5 \pm 12.3$ months; there were 18 males (48.6\%), and left arms $(67.6 \%)$ were significantly more affected than right arms $(P=0.049)$ (Table 1). The McNemar tests showed that there were significant differences between affected and normal arms in terms of the altered shape of the supinator muscle, misplacement the annular ligament in affected arms, and enlargement of the synovial fringe (Table 2).

Table 1. Baseline characteristics of patients with pulled elbow

\begin{tabular}{lcc}
\hline Variable & Study population $(n=37)$ & P-value $^{\text {a) }}$ \\
\hline Age (mo) & $28.6 \pm 12.3$ & \\
Sex (male:female) & $18(48.6): 19(51.4)$ & $>0.999$ \\
Affected arm (left:right) & $25(67.6): 12(32.4)$ & 0.049 \\
\hline
\end{tabular}

Values are presented as mean \pm SD or frequency $(\%)$.

${ }^{a}$ Chi-square test.
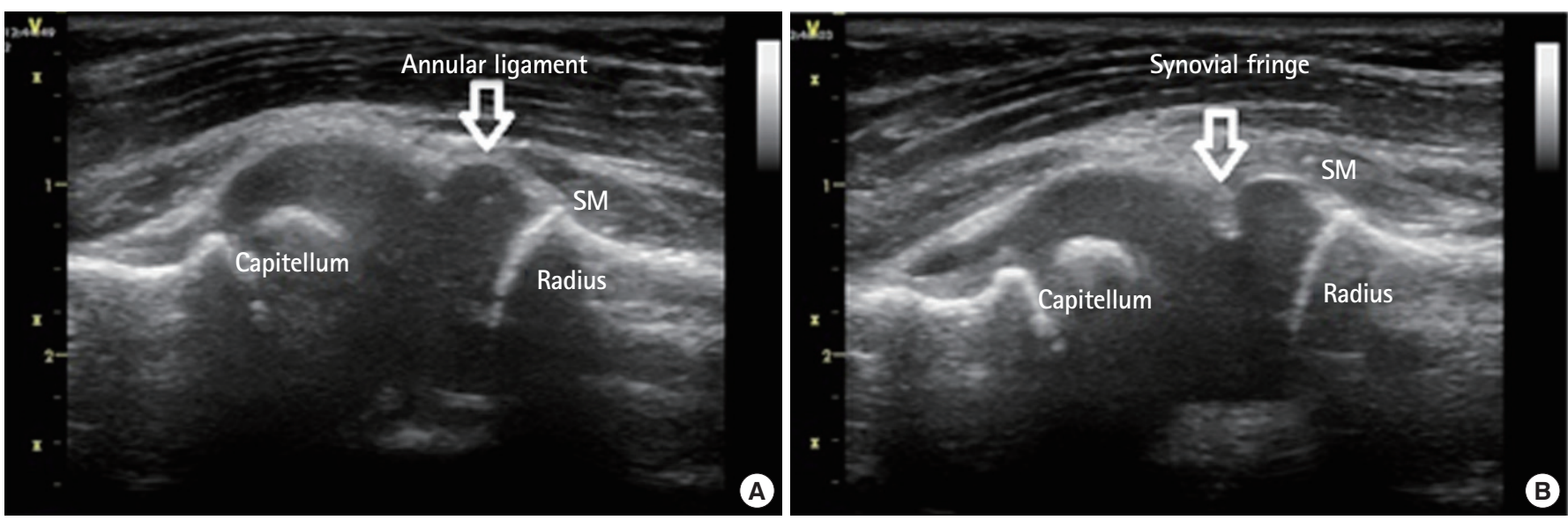

Fig. 3. Ultrasonographic findings of the radiocaptirellar joint. (A) Unaffected arm's ultrasonographic image. We can see a normal supinator muscle (SM) and synovial fringe. The annular ligament is in place on the radius (arrow). (B) Affected arm's ultrasonographic image. We can see enlargement of the synovial fringe because of entrapped annular ligament.

Table 2. Statistical differences of ultrasonographic findings

\begin{tabular}{|c|c|c|c|c|}
\hline Variable & Affected arm & Unaffected arm & 95\% Confidence interval & P-value ${ }^{\text {a) }}$ \\
\hline Change of supinator muscle shape & $34(92.9)$ & $6(16.2)$ & -4.88 to 10.34 & 0.508 \\
\hline Displacement of annular ligament & $24(64.9)$ & $0(0.0)$ & 8.89 to 17.57 & $<0.001$ \\
\hline Enlargement of synovial fringe & $23(62.2)$ & $8(21.6)$ & -5.55 to 19.5 & 0.286 \\
\hline
\end{tabular}

Values are presented as number (\%).

${ }^{\text {a) } M c N e m a r}$ test. 
Only the finding of "loss of annular ligament in place" was statistically significant $(P<0.001)$. The sensitivity, specificity, positive predictive value, and negative predictive values were 64.9\% (95\% $\mathrm{Cl}_{1} 47.5 \%$ to $79.8 \%$ ), $100.0 \%$ (95\% Cl, $90.5 \%$ to $100.0 \%$ ), $100.0 \%$ (95\% $\mathrm{Cl}, 85.8 \%$ to $100.0 \%)$, and $74.0 \%(95 \% \mathrm{Cl}, 59.7 \%$ to $85.4 \%)$, respectively.

\section{DISCUSSION}

We analyzed infants and preschool patients presenting to an urban ED with the complaint of upper extremity immobility and confirmed pulled elbow. Pulled elbow was the most common cause of upper extremity immobility in infant and preschool children, comprising 2/3 of upper extremity injuries in infants and preschool patients. ${ }^{1}$ Pulled elbow is usually caused by a traction mechanism ${ }^{7}$ in which the supinator muscle and annular ligament slip over the radial head. ${ }^{3}$ In other words, the supinator muscle is pulled proximally and entraps the radiohumeral joint. By ultrasonography, we usually find either a change in shape of the supinator muscle, the annular ligament not being over the radial head, or enlargement of the synovial fringe.

There have been attempts to diagnose pulled elbow using radiographs, with a few measurement methods, but these methods provided ambiguous results. ${ }^{8,9}$ Currently, radiographs are taken to rule out fractures. The diagnosis of pulled elbow depends on history of mechanism and physical examination. ${ }^{3}$ Reduction may be attempted without radiographs if clinical presentation is typical of a pulled elbow $7^{7,10}$ however, in case of an unclear mechanism of injury, unnecessary radiographs are often taken. ${ }^{11,12}$

We believe it would be useful to use ultrasound to confirm pulled elbow in terms of reducing unnecessary radiation, especially when the mechanism of injury is not evident or physical examination seems inconclusive. Moreover, ultrasound could be performed with the patient's arm in a neutral position, which could minimize unrecognized complications such as occult fractures. Additionally, we could reduce the length of stay in the ED and prevent patients from having to go to an X-ray room and waiting until the immobility is resolved. Unlike $X$-ray, ultrasonography is a real-time method: we could diagnose the pulled elbow, reduce the subluxation of the arm, and can confirm normalization.

Most of all, our method has a high sensitivity and specificity. Even though there have been some reports for diagnosing pulled elbow by ultrasonography, those studies have been difficult to standardize in practice. Because the studies discuss the relationship of the radius and capitellum in terms of distance, they are too vague to distinguish between normal and abnormal findings. ${ }^{3,10}$

In one recent study performed in Japan with 70 cases of pulled elbow, the researchers displayed J-shaped hypoechoic images as a pathognomonic finding of pulled elbow. They reported that the sensitivity, specificity, and accuracy of ultrasonographic diagnosis of pulled elbow were all 100\%. ${ }^{13}$ However, according to one report, pulled elbow is classified into two types of pathology. ${ }^{2}$ We also assume that there are two types of pulled elbow: total subluxation of the radial head and subtotal subluxation of the radial head. In cases with subtotal subluxation of the radial head, we could not find the J-shaped hypoechoic image as described by the previously mentioned Japanese study. One difference that could have affected the diagnostic performance was that they performed the study with one experienced physician, whereas we used two novice operators.

To the best of our knowledge, we believe that diagnosing pulled elbow by using ultrasonography may be a better technique than other methods. However, there may be a selection bias. To overcome this pitfall, a randomized controlled prospective study of patients who come to the ED with elbow pain and an unclear accidental mechanism or physical examination is further required.

In conclusion, a pulled elbow can be confirm by the ultrasonographic finding of annular ligament displacement. Because this is a significant finding, we suggest ultrasonography as a confirmative and dynamic tool for diagnosis of a pulled elbow.

\section{CONFLICT OF INTEREST}

No potential conflict of interest relevant to this article was reported.

\section{REFERENCES}

1. Schutzman SA, Teach S. Upper-extremity impairment in young children. Ann Emerg Med 1995;26:474-9.

2. Diab HS, Hamed MM, Allam Y. Obscure pathology of pulled elbow: dynamic high-resolution ultrasound-assisted classification. J Child Orthop 2010;4:539-43.

3. Kosuwon W, Mahaisavariya B, Saengnipanthkul S, Laupattarakasem $W_{1}$ Jirawipoolwon $P$. Ultrasonography of pulled elbow. J Bone Joint Surg Br 1993;75:421-2.

4. Richardson M, Kuester VG, Hoover K. The usefulness of MRI in atypical pulled/nursemaid's elbow: a case report. J Pediatr Orthop 2012;32:e20-2.

5. Kraus R, Dongowski N, Szalay G, Schnettler R. Missed elbow fractures misdiagnosed as radial head subluxations. Acta Orthop Belg 2010;76:312-5.

6. Sohn YD, Lee YS, Oh YT, Lee WW. Sonographic finding of a pulled elbow: the "hook sign". Pediatr Emerg Care 2014;30:919-21. 
7. Choung W, Heinrich SD. Acute annular ligament interposition into the radiocapitellar joint in children (nursemaid's elbow). J Pediatr Orthop 1995;15:454-6.

8. Macias CG, Wiebe R, Bothner J. History and radiographic findings associated with clinically suspected radial head subluxations. Pediatr Emerg Care 2000;16:22-5.

9. Snyder HS. Radiographic changes with radial head subluxation in children. J Emerg Med 1990;8:265-9.

10. Scapinelli R, Borgo A. Pulled elbow in infancy: diagnostic role of imaging. Radiol Med 2005;110:655-64.

11. Sacchetti A, Ramoska EE, Glascow C. Nonclassic history in children with radial head subluxations. J Emerg Med 1990;8:151-3.

12. Rudloe TF, Schutzman S, Lee LK, Kimia AA. No longer a "nursemaid's" elbow: mechanisms, caregivers, and prevention. Pediatr Emerg Care 2012;28:771-4.

13. Dohi D. Confirmed specific ultrasonographic findings of pulled elbow. J Pediatr Orthop 2013;33:829-31. 